\title{
Heterosis for Yield, Yield Components and Nutritional Traits in Mung Bean (Vigna radiata (L.) Wilczek)
}

\author{
S. Kalpana*, N.V. Naidu and D.M. Reddy \\ Department of Genetics and Plant Breeding, S. V. Agricultural College, \\ Tirupati - 517502, A.P., India \\ *Corresponding author
}

\section{A B S T R A C T}

\section{Keywords}

Heterosis, Mungbean,

Yield components,

Nutritional traits

Article Info

Accepted:

15 October 2018

Available Online:

10 November 2018
Twenty mungbean hybrids were derived five lines, four testers, through linex tester mating design were evaluated to study the magnitude of relative heterosis, heterobeltiosis and standard heterosis for yield and nutritional traits. The magnitude of relative heterosis, heterobeltiosis and standard heterosis observed for seed yield per plant were 57.45, 53.67, and 51.04 respectively. Heterosis for seed yield per plant is manifested through heterotic response for most of the yield contributing traits in additive fashion. The crosses LGG-574 x Pusa Vishal, LGG-574 x PM-5, LGG-460 x Pusa Vishal, LGG-460 x IPM-2-14 and LGG-407 x PM-5were adjudged as superior heterotic crosses for seed yield, yield components and nutritional traits. Therefore, these crosses could be exploited by adopting recurrent selection or biparental mating mating to derive high yielding segregants in mungbean.

\section{Introduction}

Greengram [Vigna radiata L. Wilczek. 2n=22] is an important pulse crop cultivated in many tropical and sub-tropical parts of the world.

Among pulses, mungbean holds a key position as a valuable short duration legume due to its wider adoptability, low input requirement and ability to improve soil fertility through nitrogen fixation. Greengram is regarded as a quality pulse due to its excellent digestibility, free from flatulence and rich protein content especially in cereal-based diet. It contains proteins $(22-28 \%)$, carbohydrates $(60-65 \%)$, fat (1-1.5\%), fibers $(3.5-4.5 \%)$, iron (40-70 $\mathrm{ppm})$ and is also a rich source of essential amino acids specially lysine, which is deficient in most of the cereals. Recently domestic consumption of greengram has increased because of the rising popularity in Indian ethnic foods and perceived health benefits (Datta et al., 2012). The productivity of greengram is low and is often related to its poor genetic makeup. Exploitation of heterosis is a quick and convenient way of combining desirable genes present in different parents into single genotype i.e. $\mathrm{F}_{1}$. The exploitation of heterosis in greengram has not been commercialized due to limited extent of out crossing. However, heterotic crosses identified could be exploited for obtaining desirable transgressive segregants for various quantitative traits in advanced generations. 


\section{Materials and Methods}

Five lines viz., TM-96-2, MGG-295, LGG574, LGG-460 and LGG-407 were crossed with each of four testers viz., Pusa Vishal, PM-5, IPM-2-14 and PM-110 in a Line $x$ Tester mating design and $20 \mathrm{~F}_{1}$ were produced during kharif, 2016. The 20 crosses along with nine parents were grown in Randomised Complete Block Design (RCBD) with three replications during rabi, 2016-17 at S.V. Agricultural College Farm, Tirupati. Each entry in each replication was grown in two rows of $3 \mathrm{~m}$ length. The spacing adopted between the rows was $30 \mathrm{~cm}$ and within a row between the plants was $10 \mathrm{~cm}$. All recommended crop production and protection practices were followed to raise a good and healthy crop. Data was recorded on five randomly selected plants in each genotype in each replication. Mean values on plant basis were recorded for traits like plant height, number of branches per plant, number of clusters per plant, number of pods per cluster, 100 seed weight, seed yield per plant while the traits days to $50 \%$ flowering and days to maturity were recorded on plot basis. Mean data of all the traits was subjected to analysis of variance as per Panse and Sukhatme (1985) to test the significance levels. Heterosis was calculated as percentage increase or decrease over mid parent, better parent and standard variety values as suggested by Rai (1979).

\section{Results and Discussion}

Analysis of variance for quantitative traits among parents and their crosses were presented in table 1 . The variance due to lines was found to be significant for all the characters except number of seeds per pod. The variance due lines $\mathrm{x}$ testers were also found to be significant for 12 characters except for the days to 50 percent flowering, and days to maturity representing the significance of non-additive variances for most of the traits. In contrary, the significance of mean sum of squares of lines and testers indicates the predominance of additive variance for respective traits. The significance of both additive and non-additive gene action for yield and its components was also reported by Barad et al., (2008) and Marappa (2008). The nature and magnitude of relative heterosis, heterobeltiosis and standard heterosis for yield attributes and nutritional traits differed among the crosses (Table 2). The results are discussed below and summarized (Table 1-5).

\section{Days to $50 \%$ flowering}

Heterosis in negative direction is considered to be desirable for this trait. Lowest mid parent heterosis of -8.30 was registered by the cross LGG-407 x PM-5, whereas highest mid parent heterosis of 12.96 was registered by LGG-460 x IPM-2-14. Out of 20 crosses only one cross LGG-407 x PM-5 exhibited significant mid parent heterosis in desirable direction. LGG407 x IPM-2-14 (9.65) registered significant highest better parent heterosis and cross LGG407 x PM-5 (-8.70) showed lowest better parent heterosis. Among the 20 crosses, none of the crosses showed significant negative better parent heterosis for this trait. On the other hand, LGG-407 x IPM-2-14 (21.36) exhibited a highest significant positive standard heterosis for days to $50 \%$ flowering, whereas lowest magnitude of standard heterosis was recorded by LGG-574 x Pusa Vishal (1.94) and LGG-407x PM-5 (1.94). None of the 20 crosses showed significant negative standard heterosis in desirable direction.

\section{Days to maturity}

Relative heterosis for this trait varied from 5.85 to 4.67 , where in the highest relative heterosis was recorded by the cross LGG574× IPM-2-14(4.67) and lowest by LGG-407 
$\times$ PM-5 (-5.85). Among the crosses heterobeltiosis varied from -7.21 to 3.40 . The cross LGG-574× IPM-2-14 (3.40) registered highest significant positive better parent heterosis and LGG-407 $\times$ PM-5 (-7.21) recorded lowest significant negative better parent heterosis. Standard heterosis among the crosses for this trait ranged from -1.03 to 9.74. High standard heterosis was exhibited by TM96-2 $\times$ Pusa Vishal (9.74) and the low standard heterosis by LGG-407 × PM-5 (1.03) in desirable direction hence this cross can be exploited to evolve short duration genotypes.

\section{Plant height}

Among the crosses the relative heterosis ranged from -11.36 (LGG-407×Pusa Vishal) to 32.64 (TM-96-2 $\times$ PM-110). Positive significant relative heterosis was registered by TM-96-2 × PM-110 (32.64) followed by TM96-2 $\times$ Pusa Vishal (28.39) and MGG-295 $\times$ Pusa Vishal (26.40). Significant relative heterosis in negative direction was recorded by LGG-407 $\times$ Pusa Vishal (-11.36). Ten out of 20 crosses exhibited significant positive relative heterosis for this trait in contrary only two crosses exhibited significant negative relative heterosis. The magnitude of heterobeltiosis among the crosses for this trait varied from -24.98 to 30.87 . The cross TM96-2 × PM-110 (30.87) registered high significant positive heterobeltiosis followed by TM-96-2 $\times$ Pusa Vishal (27.12) and MGG-295 $\times$ Pusa Vishal (22.49). Out of 20 crosses, seven crosses recorded significant negative better parent heterosis on the other hand seven crosses displayed significant positive heterosis over better parent. The range of standard heterosis varied from -20.88 to 7.13. Cross LGG-407 × PM-5 (7.13) registered highly significant positive standard heterosis followed by LGG-460 × IPM2-14 (4.30) and the low standard heterosis was recorded by cross LGG-574× IPM-2-14 (-20.88) The crosses TM-96-2 × PM-110, TM-96-2 $\times$ Pusa Vishal, MGG-295 $\times$ Pusa Vishal, MGG-295 $\times$ PM-5 registered significant positive relative heterosis, heterobeltiosis and standard heterosis. Hence, these crosses could be effectively exploited in breeding programmes for obtaining segregants with increased plant height.

\section{Number of branches per plant}

The maximum relative heterosis for this trait was registered by TM-96-2 $\times$ PM-110 (110.77) followed by TM-96-2 $\times$ Pusa Vishal (87.70) and LGG-460 $\times$ Pusa Vishal (82.66) while low heterosis over mid parent was recorded by LGG-574 × IPM-2-14 (-44.72).

Among 20 crosses studied seven crosses showed significant positive relative heterosis and nine crosses recorded significant negative relative heterosis for number of branches per plant. High significant positive heterobeltiosis was displayed by TM-96-2 × Pusa Vishal (82.39), whereas LGG-407 $\times$ Pusa Vishal (64.30) recorded significant negative heterobeltiosis. Among 20 crosses evaluated seven crosses exhibited significant positive heterobeltiosis, whereas 12 crosses showed significant negative heterosis over better parent. Standard heterosis for this trait varied from -52.78 (LGG-574 $\times$ IPM-2-14) to 31.67 (LGG-460 $\times$ Pusa Vishal).

Significant positive heterosis over standard variety was registered by the cross LGG-460 $\times$ Pusa Vishal (31.67). Among the 20 crosses studied, five crosses exhibited significant positive standard heterosis Crosses LGG-574 $\times$ Pusa Vishal, LGG-574 × PM-5, LGG-460 × Pusa Vishal and LGG-460 $\times$ IPM-2-14 recorded high significant relative heterosis, heterobeltiosis and standard heterosis. Hence, these crosses can be employed in further breeding programmes to derive the segregants with more number of branches per plant. 


\section{Number of clusters per plant}

Relative heterosis for this trait varied from 11.53 (LGG-574 $\times$ IPM-2-14) to 172.36 (TM96-2× PM-110). Out of 20 crosses, 15 crosses registered significant positive relative heterosis while only one cross recorded significant negative relative heterosis. Heterobeltiosis ranged from -36.32 (LGG-574 $\times$ IPM-2-14) to 169.40 (TM-96-2× PM-110). Among 20 crosses, 13 crosses exhibited significant heterobeltiosis in positive direction, while three crosses exhibited significant heterobeltiosis in negative direction. Standard heterosis over standard parent LGG-460 for number of clusters per plant varied from $-53.56($ MGG-295 $\times$ PM110) to 47.29 (LGG-574 $\times$ PM-5). The crosses TM-96-2 × IPM-2-14, TM-96-2 × PM-110, MGG-295 $\times$ Pusa Vishal, LGG-574 $\times$ Pusa Vishal, LGG-574 $\times$ PM-5, LGG-460 $\times$ Pusa Vishal, LGG-460 $\times$ PM-5, LGG-460 $\times$ IPM214, LGG-460 $\times$ PM-110, LGG-407 x PM-5 and LGG-407 x IPM-2-14 also registered significant positive heterosis over standard variety, mid parent and better parent. Hence, these crosses could be considered as heterotic cross combinations for this particular trait.

\section{Number of pods per cluster}

Low relative heterosis was recorded for the cross LGG-574× PM-110(2.86). In contrast high relative heterosis was observed in the cross TM-96-2 × IPM2-14 (37.51). Out of twenty crosses, 14 crosses displayed significant positive relative heterosis and none of the crosses registered significant negative relative heterosis for this trait. High heterobeltiosis for number of pods per cluster was exhibited by TM-96-2 $\times$ IPM-2-14 (31.59) and the cross LGG-574 $\times$ PM-110 recorded lowest percent of heterobeltiosis (-6.98). Eleven out of 20 crosses displayed significant positive heterosis over better parent. The range of heterosis over standard variety varied from -11.25 (MGG-295 × PM$110)$ to 18.97 (LGG-460 $\times$ IPM-2-14). Four crosses exhibited significant positive heterosis over standard variety, where as one cross showed significant negative standard heterosis. The crosses TM-96-2 $\times$ IPM-2-14, LGG-574 $\times$ Pusa Vishal and LGG-460 $\times$ IPM2-14 and LGG-407 x Pusa Vishal showed significant positive relative heterosis and heterobeltiosis and standard heterosis. These crosses are of worth importance for evolving genotypes with improved number of pods per cluster subsequently aiding in the improvement of seed yield in greengram.

\section{Number of seeds per pod}

Among the 20 crosses, the relative heterosis ranged from -6.59 (LGG-407 $\times$ PM-110) to 22.48 (LGG-460 $\times$ Pusa Vishal). Ten out of 20 crosses recorded significant positive relative heterosis, while none of the cross recorded negative relative heterosis.

Low significant heterobeltiosis was observed in LGG-574 $\times$ PM-110 (-9.77), whereas the cross MGG-295 $\times$ PM-110 (15.40) recorded high significant heterobeltiosis for this trait. Out of 20 crosses, five crosses registered significant heterobeltiosis in positive direction and two crosses displayed significant heterobeltosis in negative direction.

The percent heterosis over standard variety ranged from -8.15 (LGG-407 $\times$ PM-110) to 14.14 (LGG-574 $\times$ Pusa Vishal). Five out of 20 crosses displayed significant positive standard heterosis. The crosses MGG-295 $\times$ IPM-2-14, MGG-295 × PM-110, LGG-460 $\times$ Pusa Vishal, LGG-460 $\times$ IPM-2-14exhibited significant positive standard heterosis along with significant positive relative heterosis and heterobeltiosis. Therefore, these crosses can be adequately exploited in breeding programmes to increase the yield of greengram. 
Table.1 Analysis of variance for Line $\mathrm{x}$ Tester analysis for different quantitative traits in mungbean

\begin{tabular}{|c|c|c|c|c|c|c|c|c|c|c|c|c|c|c|c|}
\hline Source & df. & $\begin{array}{c}\text { Days to } \\
50 \% \\
\text { flowering }\end{array}$ & $\begin{array}{l}\text { Days to } \\
\text { maturity }\end{array}$ & $\begin{array}{c}\text { Plant } \\
\text { height } \\
(\mathrm{cm})\end{array}$ & $\begin{array}{c}\text { Number } \\
\text { of } \\
\text { branches } \\
\text { plant }^{-1}\end{array}$ & $\begin{array}{c}\text { Number } \\
\text { of } \\
\text { clusters } \\
\text { plant }^{-1}\end{array}$ & $\begin{array}{l}\text { Number } \\
\text { of pods } \\
\text { cluster }{ }^{-1}\end{array}$ & $\begin{array}{c}\text { Number } \\
\text { of seeds } \\
\text { pod }^{-1}\end{array}$ & $\begin{array}{l}100 \text { seed } \\
\text { weight } \\
\text { (g) }\end{array}$ & $\begin{array}{c}\text { Seed } \\
\text { yield } \\
\text { plant }^{-1} \\
(\mathrm{~g})\end{array}$ & $\begin{array}{c}\text { Harvest } \\
\text { index } \\
(\%)\end{array}$ & $\begin{array}{c}\text { Total } \\
\text { protein } \\
\text { content }(\%)\end{array}$ & $\begin{array}{l}\text { Total } \\
\text { sugars } \\
(\%)\end{array}$ & $\begin{array}{c}\text { Reducing } \\
\text { sugars } \\
(\%)\end{array}$ & $\begin{array}{l}\text { Non-reducing } \\
\text { sugars (\%) }\end{array}$ \\
\hline Replications & 2 & 4.63 & 1.53 & 8.30 & 0.08 & 0.63 & 0.10 & 0.09 & 0.21 & 1.70 & 4.10 & 0.11 & 0.13 & 0.01 & 0.18 \\
\hline Lines & 4 & $11.83^{* *}$ & $19.10 * *$ & $150.69^{* *}$ & $3.08^{*}$ & $20.01 * *$ & $0.39 * *$ & 0.52 & $2.45^{* *}$ & $7.80^{*}$ & $35.45^{* *}$ & $1.49 * *$ & $0.47 * *$ & $0.01 * *$ & $0.44 * *$ \\
\hline Testers & 3 & 0.31 & $2.97^{*}$ & $17.06^{* *}$ & 0.61 & 0.08 & $0.04^{*}$ & $0.72 * *$ & $0.30 * *$ & $1.68^{*}$ & $8.57 * *$ & $2.45^{* *}$ & $0.57 * *$ & $0.01 * *$ & $0.71 * *$ \\
\hline Lines $\times$ Testers & 12 & 7.47 & 7.48 & $58.84 * *$ & $1.36^{* *}$ & $24.87 * *$ & $0.20 * *$ & $1.34 * *$ & $1.34 * *$ & $19.01^{* *}$ & $65.33^{* *}$ & $3.45^{* *}$ & $1.50 * *$ & $0.02 * *$ & $1.62 * *$ \\
\hline Error & 56 & 4.91 & 4.73 & 4.24 & 0.03 & 0.18 & 0.05 & 0.29 & 0.07 & 2.20 & 7.39 & 0.17 & 0.03 & 0.01 & 0.05 \\
\hline
\end{tabular}

Table.2 Estimates of Relative Heterosis (RH), Heterobeltiosis (HB) and Standard Heterosis (SH) for days to 50\% flowering, days to maturity and plant height in greengram

\begin{tabular}{|c|c|c|c|c|c|c|c|c|c|c|}
\hline \multirow{2}{*}{\multicolumn{2}{|c|}{ Crosses }} & \multicolumn{3}{|c|}{ Days to $50 \%$ flowering } & \multicolumn{3}{|c|}{ Days to maturity } & \multicolumn{3}{|c|}{ Plant height (cm) } \\
\hline & & RH & HB & SH & RH & HB & SH & RH & HB & SH \\
\hline TM-96-2 × Pusa Vishal & & 1.72 & -0.84 & $14.56^{* *}$ & 2.39 & 0.01 & $9.74 * *$ & $28.39^{* *}$ & $27.12 * *$ & $2.10^{*}$ \\
\hline TM-96-2 × PM-5 & & -0.85 & -2.52 & $12.62 *$ & -0.95 & -2.34 & $7.18^{*}$ & $13.16^{* *}$ & $8.56^{*}$ & $-6.98^{*}$ \\
\hline TM-96-2 × IPM-2-14 & & -0.86 & -3.36 & $11.65^{*}$ & -0.48 & -2.34 & $7.18^{*}$ & 1.47 & -5.38 & $-13.87 * *$ \\
\hline TM-96-2 × PM-110 & & 1.29 & -0.84 & $14.56^{* *}$ & -3.06 & -3.74 & $5.64^{*}$ & $32.64 * *$ & $30.87 * *$ & $5.84^{*}$ \\
\hline MGG-295 $\times$ Pusa Vishal & & 3.60 & 1.77 & $11.65^{*}$ & 0.97 & -0.48 & $7.18^{*}$ & $26.40^{* *}$ & $22.49 * *$ & $4.87 *$ \\
\hline MGG-295 $\times$ PM-5 & & 6.25 & 3.48 & $15.53^{* *}$ & 0.00 & -0.48 & $7.18^{*}$ & $17.01^{* *}$ & $16.96^{* * *}$ & $0.22 *$ \\
\hline MGG-295 × IPM-2-14 & & 4.50 & 2.65 & $12.62 *$ & 1.92 & 0.95 & $8.72 * *$ & 1.52 & -1.50 & $-10.34 * *$ \\
\hline MGG-295 $\times$ PM-110 & & 4.04 & 1.75 & $12.62 *$ & -0.71 & -0.95 & $7.18^{*}$ & -2.50 & -5.20 & $-18.84 * *$ \\
\hline LGG-574 $\times$ Pusa Vishal & & -5.83 & -7.08 & 1.94 & -1.73 & -2.45 & 2.05 & $19.90 * *$ & $15.76^{* * *}$ & -0.13 \\
\hline LGG-574 $\times$ PM-5 & & -4.89 & -6.96 & 3.88 & 0.73 & -0.96 & $5.64 *$ & $13.76^{* *}$ & $13.37 * *$ & -2.18 \\
\hline LGG-574 × IPM-2-14 & & 3.14 & 1.77 & $11.65^{*}$ & $4.67 *$ & $3.40^{*}$ & $9.23 *$ & $-10.75 * *$ & $-13.08 * *$ & $-20.88 * *$ \\
\hline LGG-574 $\times$ PM-110 & & 2.68 & 0.88 & $11.65^{*}$ & -2.43 & -4.74 & 3.08 & -3.72 & -6.73 & $-19.53 * *$ \\
\hline LGG-460 $\times$ Pusa Vishal & & 4.63 & -0.00 & 9.71 & 1.75 & -0.49 & 4.10 & 18.59 ** & 6.92 & 6.92 \\
\hline LGG-460x PM-5 & & 6.42 & 0.87 & $12.62 *$ & 3.72 & 0.48 & $7.18^{*}$ & -1.40 & $-8.46^{*}$ & $-8.46^{*}$ \\
\hline LGG-460 × IPM-2-14 & & $12.96^{* *}$ & 7.96 & $18.45^{* *}$ & 1.75 & -0.97 & 4.62 & $9.20 * *$ & 4.30 & $4.30 *$ \\
\hline LGG-460 $\times$ PM-110 & & $8.76^{*}$ & 3.51 & $14.56^{* *}$ & 0.00 & -3.79 & 4.10 & -1.25 & $-10.69 * *$ & $-10.69 * *$ \\
\hline LGG-407 $\times$ Pusa Vishal & & 7.49 & 7.02 & $18.45^{* *}$ & 1.97 & 1.47 & $6.15^{*}$ & $-11.36 * *$ & $-24.98 * *$ & $-13.02 * *$ \\
\hline LGG-407 $\times$ PM-5 & & $-8.30 *$ & -8.70 & 1.94 & $-5.85^{*}$ & $-7.21 * *$ & $-1.03 *$ & $6.25^{*}$ & $-7.61^{*}$ & $7.13^{*}$ \\
\hline LGG-407 xIPM-2-14 & & $10.13^{*}$ & $9.65^{*}$ & $21.36^{* *}$ & 0.98 & 0.00 & $5.64 *$ & -5.77 & $-15.90 * *$ & -2.48 \\
\hline LGG-407 × PM-110 & & 5.26 & 5.26 & $16.50 * *$ & -0.24 & -2.37 & $5.64 *$ & -5.00 & $-19.37 * *$ & -6.50 \\
\hline S.E.(Heterosis) & & 1.56 & 1.80 & 1.80 & 1.53 & 1.77 & 1.77 & 1.45 & 1.68 & 1.68 \\
\hline Range & Low & -8.30 & -8.70 & 1.94 & -5.85 & -7.21 & -1.03 & -11.36 & -24.98 & -20.88 \\
\hline & High & 12.96 & 9.65 & 21.36 & 4.67 & 3.40 & 9.74 & 32.64 & 30.87 & 7.13 \\
\hline
\end{tabular}

RH: Relative heterosis; HB: Heterobeltiosis; SH: Standard heterosis; * Significant at 5\% level; ** Significant at $1 \%$ level 
Table.3 Estimates of Relative Heterosis (RH), Heterobeltiosis (HB) and Standard Heterosis (SH) for number of branchesplant ${ }^{-1}$, number of clusters plant ${ }^{-1}$ and number of pods cluster ${ }^{-1}$ in greengram

\begin{tabular}{|c|c|c|c|c|c|c|c|c|c|}
\hline \multirow[t]{2}{*}{ Crosses } & \multicolumn{3}{|c|}{ Number of branches plant ${ }^{-1}$} & \multicolumn{3}{|c|}{ Number of clusters plant ${ }^{-1}$} & \multicolumn{3}{|c|}{ Number of pods cluster ${ }^{-1}$} \\
\hline & RH & HB & SH & RH & HB & SH & RH & HB & SH \\
\hline TM-96-2 $\times$ Pusa Vishal & $87.70 * *$ & $82.39 * *$ & $-19.44 * *$ & 9.23 & 3.77 & $-45.05 * *$ & $28.94 * *$ & $27.18 * *$ & 5.72 \\
\hline TM-96-2 × PM-5 & $56.32 * *$ & $19.30 * *$ & -5.56 & 14.56 & 12.85 & $-44.56 * *$ & $21.29 * *$ & $18.38 * *$ & 0.54 \\
\hline TM-96-2 × PM-110 & $110.77 * *$ & $66.54 * *$ & $19.58 * *$ & $172.36 * *$ & $169.40 * *$ & $31.24 * *$ & $25.29 * *$ & $25.22 * *$ & 1.36 \\
\hline $\begin{array}{l}\text { MGG-295 } \times \text { Pusa } \\
\text { Vishal }\end{array}$ & 8.93 & $-16.67 *$ & $-30.56 * *$ & $158.48 * *$ & $153.31 * *$ & $34.13 * *$ & $23.88 * *$ & $22.05 * *$ & 1.45 \\
\hline MGG-295 $\times$ IPM-2-14 & $-42.76 * *$ & $-44.13 * *$ & $-51.11 * *$ & $101.30 * *$ & $98.08 * *$ & 0.69 & 8.58 & 3.79 & -8.17 \\
\hline MGG-295 $\times$ PM-110 & $-33.75 * *$ & $-38.33 * *$ & $-48.61 * *$ & -6.71 & -8.65 & $-53.56 * *$ & 9.83 & 9.64 & $-11.25^{*}$ \\
\hline LGG-574 $\times$ Pusa Vishal & $73.42 * *$ & $32.67 * *$ & $10.56^{*}$ & $62.75^{* *}$ & $19.87 * *$ & $34.18 * *$ & $23.33 * *$ & $12.87 *$ & $12.98^{*}$ \\
\hline LGG-574 x PM-5 & $59.32 * *$ & $55.33 * *$ & $29.44 * *$ & $82.90 * *$ & $31.59 * *$ & $47.29 * *$ & $9.76^{*}$ & 1.45 & 1.54 \\
\hline LGG-574 x IPM-2-14 & $-44.72 * *$ & $-46.03 * *$ & $-52.78 * *$ & $-11.53 *$ & $-36.32 * *$ & $-28.72 * *$ & 6.64 & 0.45 & 0.54 \\
\hline LGG-574 × PM-110 & $-38.05 * *$ & $-42.33 * *$ & $-51.94 * *$ & 3.25 & $-25.91 * *$ & $-17.07 * *$ & 2.86 & -6.98 & -6.90 \\
\hline LGG-460 $\times$ PM-110 & -10.27 & $-22.92 * *$ & $-22.92 * *$ & $92.44 * *$ & $43.10 * *$ & $43.10 * *$ & $19.56 * *$ & 8.17 & 8.17 \\
\hline LGG-407 x Pusa Vishal & $-44.17 * *$ & $-64.30 * *$ & $-43.47 * *$ & $33.77 * *$ & 0.00 & 6.97 & $31.77 * *$ & $28.75 * *$ & $12.16^{*}$ \\
\hline LGG-407 x PM-5 & $-12.28 * *$ & $-34.21 * *$ & 4.17 & $73.33 * *$ & $26.47 * *$ & $35.27 * *$ & $21.10 * *$ & $19.58 * *$ & 4.17 \\
\hline LGG-407 × IPM-2-14 & $-30.06 * *$ & $-45.70 * *$ & $-14.03 *$ & $80.96 * *$ & $32.10 * *$ & $41.30 * *$ & 7.60 & 6.77 & -5.54 \\
\hline LGG-407 × PM-110 & $-39.89 * *$ & $-56.32 * *$ & $-30.83 * *$ & $12.14^{*}$ & $-18.39 * *$ & $-12.71 * *$ & $15.87 * *$ & $11.77 *$ & -2.63 \\
\hline S.E.(Heterosis) & 0.11 & 0.12 & 0.12 & 0.30 & 0.34 & 0.34 & 0.16 & 0.18 & 0.18 \\
\hline Range Low & -44.72 & -64.30 & -52.78 & -11.53 & -36.32 & -53.56 & 2.86 & -6.98 & -11.25 \\
\hline High & 110.77 & 82.39 & 31.67 & 172.36 & 169.40 & 47.29 & 37.51 & 31.59 & 18.97 \\
\hline
\end{tabular}

RH: Relative heterosis; HB: Heterobeltiosis; SH: Standard heterosis

*Significant at 5\%level; ** Significant at $1 \%$ level 
Table.4 Estimates of Relative Heterosis (RH), Heterobeltiosis (HB) and Standard Heterosis (SH) for numberof seeds pod ${ }^{-1}, 100$ seed $^{-1}$ weight, seed yield plant ${ }^{-1}$ and harvest index in greengram

\begin{tabular}{|c|c|c|c|c|c|c|c|c|c|c|c|c|}
\hline \multirow[t]{2}{*}{ Crosses } & \multicolumn{3}{|c|}{ Number of seeds per pod } & \multicolumn{3}{|c|}{100 Seed weight (g) } & \multicolumn{3}{|c|}{ Seed yield per plant (g) } & \multicolumn{3}{|c|}{ Harvest index (g) } \\
\hline & RH & HB & SH & RH & HB & SH & RH & HB & SH & RH & HB & SH \\
\hline $\begin{array}{l}\text { TM-96-2 } \times \text { Pusa } \\
\text { Vishal }\end{array}$ & $8.93 *$ & 1.43 & 1.17 & 2.11 & -6.85 & 2.98 & 7.34 & 6.60 & -1.84 & $-12.96^{*}$ & $-16.83 * *$ & $-14.36^{*}$ \\
\hline TM-96-2 × PM-5 & 5.76 & 1.53 & 1.27 & $-12.03 *$ & $-17.50 * *$ & $-14.13 *$ & $57.45 * *$ & $53.67 * *$ & $39.55 * *$ & 10.14 & 10.01 & 3.20 \\
\hline TM-96-2 x IPM-2-14 & -1.77 & -3.66 & -3.90 & $16.62 * *$ & $14.33 *$ & 8.51 & $46.48 * *$ & $44.99 * *$ & $31.67 * *$ & $22.67 * *$ & $21.66 * *$ & $16.04 * *$ \\
\hline TM-96-2 × PM-110 & $8.64 *$ & 6.52 & 6.25 & -4.22 & $-13.53 * *$ & -2.13 & $35.05 * *$ & 24.01 & 12.61 & $-18.19 * *$ & $-18.33 * *$ & $-23.13 * *$ \\
\hline $\begin{array}{l}\text { MGG-295 } \times \text { Pusa } \\
\text { Vishal } \\
\end{array}$ & 6.93 & 1.29 & -2.60 & -6.07 & $-11.24^{*}$ & -1.87 & $45.03 * *$ & $30.44 *$ & 20.12 & $11.10^{*}$ & 3.65 & 6.72 \\
\hline MGG-295 $\times$ PM-5 & $12.91 * *$ & $10.32 *$ & 6.09 & $-11.06^{*}$ & $-13.49 *$ & -9.96 & 14.87 & 6.30 & -8.10 & $11.01 *$ & 8.37 & 1.43 \\
\hline $\begin{array}{l}\text { MGG-295 × IPM-2- } \\
14\end{array}$ & $13.44 * *$ & $13.29 * *$ & $8.94 *$ & $32.88 * *$ & $30.54 * *$ & $28.43 * *$ & $45.47 * *$ & $32.87 *$ & 18.22 & 10.19 & 6.59 & 1.67 \\
\hline MGG-295 $\times$ PM-110 & $15.59 * *$ & $15.40 * *$ & $10.97 * *$ & $34.35 * *$ & $25.56 * *$ & $42.13 * *$ & 10.19 & 8.45 & -17.63 & $16.49 * *$ & $13.41^{*}$ & 6.74 \\
\hline $\begin{array}{l}\text { LGG-574 } \times \text { Pusa } \\
\text { Vishal }\end{array}$ & $18.21 * *$ & 6.57 & $14.14 * *$ & $7.89 *$ & -6.10 & $40.17 * *$ & $47.98 * *$ & $34.80 * *$ & $51.04 * *$ & 8.77 & 4.07 & $17.30 * *$ \\
\hline LGG-574 × PM-5 & 3.22 & -4.17 & 2.63 & $-8.36^{*}$ & $-22.24 * *$ & $16.09 * *$ & $43.83 * *$ & $27.40 * *$ & $47.56 * *$ & $11.93 *$ & 2.43 & $15.46^{* *}$ \\
\hline LGG-574 $\times$ IPM-2-14 & -0.11 & -5.33 & 1.40 & $-23.46^{* *}$ & $-37.40 * *$ & -6.55 & $-22.39 *$ & $-30.38 * *$ & -21.99 & 4.74 & -3.31 & 8.98 \\
\hline LGG-574 x PM-110 & -4.76 & $-9.77 *$ & -3.36 & $-27.63 * *$ & $-36.37 * *$ & -5.02 & 0.81 & -15.43 & -5.23 & 2.59 & -5.87 & 6.10 \\
\hline $\begin{array}{l}\text { LGG-460 } \times \text { Pusa } \\
\text { Vishal }\end{array}$ & $22.48 * *$ & $13.92 * *$ & $13.92 * *$ & -1.76 & -6.45 & 3.40 & $53.63 * *$ & $47.56 * *$ & $42.76^{* *}$ & $10.98 *$ & 9.38 & $12.62 *$ \\
\hline LGG-460x PM-5 & 6.88 & 2.47 & 2.47 & -5.51 & -7.36 & -3.57 & 14.62 & 6.85 & 6.85 & $-22.82 * *$ & $-25.29 * *$ & $-25.29 * *$ \\
\hline LGG-460 $\times$ IPM-2-14 & $11.09 * *$ & $8.81 *$ & $8.81 *$ & 4.01 & 1.36 & 1.36 & $49.01 * *$ & $40.80 * *$ & $40.80 * *$ & $13.95 * *$ & 11.32 & 11.32 \\
\hline LGG-460 $\times$ PM-110 & 4.48 & 2.31 & 2.31 & $-19.52 * *$ & $-24.21 * *$ & $-14.21 *$ & $48.28 * *$ & $30.46 * *$ & $30.46 * *$ & 4.60 & 1.53 & 1.53 \\
\hline $\begin{array}{l}\text { LGG-407 } \times \text { Pusa } \\
\text { Vishal }\end{array}$ & $9.66 *$ & 1.60 & 2.44 & $-11.01 *$ & $-11.62 *$ & -2.30 & 5.21 & -1.79 & 4.33 & 8.95 & 7.55 & 10.73 \\
\hline LGG-407 × PM-5 & $9.09 *$ & 4.18 & 5.04 & -7.49 & -9.59 & -1.45 & $36.87 * *$ & $24.13^{*}$ & $31.86^{* *}$ & $13.83 * *$ & 10.01 & 10.36 \\
\hline LGG-407 $\times$ IPM-2-14 & 6.17 & 3.58 & 4.44 & $-13.86 * *$ & $-19.44 * *$ & $-12.17 *$ & $24.89^{*}$ & 14.75 & 21.89 & 4.35 & 1.78 & 2.11 \\
\hline LGG-407 $\times$ PM-110 & -6.59 & $-8.90 *$ & -8.15 & $-11.22 *$ & $-12.86 * *$ & -1.36 & -13.33 & $-25.68 *$ & -21.05 & 2.31 & -0.86 & -0.53 \\
\hline S.E.(Heterosis) & 0.37 & 0.43 & 0.43 & 0.18 & 0.21 & 0.21 & 1.04 & 1.21 & 1.21 & 1.92 & 2.21 & 2.21 \\
\hline Range Low & -6.59 & -9.77 & -8.15 & -27.63 & -37.40 & -14.21 & -22.39 & -30.38 & -21.99 & -22.82 & -25.29 & -25.29 \\
\hline High & 22.48 & 15.40 & 14.14 & 34.35 & 30.54 & 42.13 & $\mathbf{5 7 . 4 5}$ & 53.67 & 51.04 & 22.67 & 21.66 & 17.30 \\
\hline
\end{tabular}

RH: Relative heterosis; HB: Heterobeltiosis; SH: Standard heterosis

* Significant at $5 \%$ level; ** Significant at $1 \%$ level 
Table.5 Estimates of Relative Heterosis (RH), Heterobeltiosis (HB) and Standard Heterosis (SH) for total protein content, total sugars, reducing sugars and non-reducing sugars in greengram

\begin{tabular}{|c|c|c|c|c|c|c|c|c|c|c|c|c|}
\hline \multirow[t]{2}{*}{ Crosses } & \multicolumn{3}{|c|}{ Total protein content $(\%)$} & \multicolumn{3}{|c|}{ Total sugars (\%) } & \multicolumn{3}{|c|}{ Reducing sugars (\%) } & \multicolumn{3}{|c|}{ Non-reducing sugars (\%) } \\
\hline & RH & HB & SH & RH & HB & SH & RH & HB & SH & RH & HB & SH \\
\hline $\begin{array}{l}\text { TM-96-2 } \times \text { Pusa } \\
\text { Vishal }\end{array}$ & $4.40^{* *}$ & 4.14 & -1.57 & $-13.26 * *$ & $-14.39 * *$ & $-20.99 * *$ & -2.34 & $-7.39 * *$ & $-16.81 * *$ & $-14.25 * *$ & $-15.06 * *$ & $-21.44 * *$ \\
\hline TM-96-2 $\times$ PM-5 & 1.40 & -1.77 & -1.47 & $4.62 * *$ & -0.66 & 1.96 & $10.17 * *$ & $5.91 * *$ & 3.10 & $-17.14 * *$ & $-21.42 * *$ & $-18.94 * *$ \\
\hline TM-96-2 $\times$ IPM-2-14 & $-6.24 * *$ & $-9.77 * *$ & $-8.25 * *$ & $-11.07 * *$ & $-13.20 * *$ & $-15.87 * *$ & -3.23 & -3.94 & $-13.72 * *$ & $-14.62 * *$ & $-19.35^{* *}$ & $-16.12 * *$ \\
\hline TM-96-2 × PM-110 & $-5.06 * *$ & $-5.79 * *$ & $-10.03 * *$ & 1.45 & -0.13 & $-4.88 * *$ & $9.45 * *$ & $8.37 * *$ & -2.65 & 1.20 & -0.14 & $-5.14^{*}$ \\
\hline $\begin{array}{l}\text { MGG-295 } \times \text { Pusa } \\
\text { Vishal } \\
\end{array}$ & $-6.65 * *$ & $-8.45^{* *}$ & $-10.00 * *$ & $-25.50 * *$ & $-27.73 * *$ & $-30.91 * *$ & 3.83 & 3.26 & $-15.93 * *$ & $-28.00 * *$ & $-30.31 * *$ & $-32.43^{* *}$ \\
\hline MGG-295 $\times$ PM-5 & $-5.60 * *$ & $-6.54 * *$ & $-6.25 * *$ & $-5.04 * *$ & $-8.30 * *$ & $-5.88 * *$ & $-22.77 * *$ & $-29.09 * *$ & $-30.97 * *$ & -1.73 & $-4.69 *$ & -1.67 \\
\hline $\begin{array}{l}\text { MGG-295 × IPM-2- } \\
14\end{array}$ & $-12.78 * *$ & $-14.23 * *$ & $-12.78 * *$ & $-14.14 * *$ & $-14.73 * *$ & $-17.35^{* *}$ & $18.75^{* *}$ & $14.00 * *$ & 0.88 & $-18.67 * *$ & $-21.42 * *$ & $-18.28 * *$ \\
\hline MGG-295 $\times$ PM-110 & $-5.17 * *$ & $-6.53 * *$ & $-8.10 * *$ & $-11.46^{* *}$ & $-11.63 * *$ & $-15.51 * *$ & $18.02 * *$ & $13.57 * *$ & 0.00 & $-13.62 * *$ & $-14.50 * *$ & $-17.09 * *$ \\
\hline $\begin{array}{l}\text { LGG-574 } \times \text { Pusa } \\
\text { Vishal }\end{array}$ & 1.80 & -0.68 & -1.32 & 2.13 & -1.85 & $-4.32 *$ & $12.34 * *$ & $7.54 * *$ & $-5.31 *$ & -0.63 & -4.55 & $-5.98^{*}$ \\
\hline LGG-574 × PM-5 & $5.27 * *$ & $5.72 * *$ & $5.43^{* *}$ & $-6.93 * *$ & $9.27 * *$ & $-6.88 * *$ & $4.06^{*}$ & -0.91 & -3.54 & $-8.02 * *$ & $-10.09 * *$ & $7.25 * *$ \\
\hline LGG-574 x IPM-2-14 & $-13.19 * *$ & $-14.19 * *$ & $-12.74 * *$ & -1.81 & -2.09 & $-4.56^{*}$ & $10.78 * *$ & $10.50 * *$ & -2.21 & -3.41 & $-5.96^{*}$ & -2.20 \\
\hline LGG-574 × PM-110 & $-9.66 * *$ & $-11.41 * *$ & $-11.99 * *$ & $-7.18 * *$ & $-8.24 * *$ & $-10.56^{* *}$ & $-10.55^{* *}$ & $-10.55 * *$ & $-21.24 * *$ & $-10.31 * *$ & $-11.91 * *$ & $-13.22 * *$ \\
\hline $\begin{array}{l}\text { LGG-460 } \times \text { Pusa } \\
\text { Vishal }\end{array}$ & 1.59 & -1.20 & -1.19 & $4.70 * *$ & -0.60 & -0.60 & $-21.57 * *$ & $-29.20 * *$ & $29.20 * *$ & $4.40 *$ & -0.44 & -0.44 \\
\hline LGG-460x PM-5 & $-11.94 * *$ & $-12.08 * *$ & $-11.81 * *$ & $-11.01 * *$ & $12.15 * *$ & $-9.84 * *$ & $-12.56^{* *}$ & $-13.72 * *$ & $-13.72 * *$ & $-10.90 * *$ & $-12.27 * *$ & $9.49 * *$ \\
\hline LGG-460 × IPM-2-14 & $3.85 * *$ & $4.65 * *$ & $3.04 *$ & $15.61 * *$ & $16.91 * *$ & $16.91 * *$ & $2.82 *$ & $8.41 * *$ & $8.41 * *$ & 0.07 & -1.86 & $2.07 *$ \\
\hline LGG-460 × PM-110 & $-12.58 * *$ & $-14.55^{* *}$ & $-14.55^{* *}$ & $-15.71 * *$ & $-17.71 * *$ & $-17.71 * *$ & $-19.06 * *$ & $-23.89 * *$ & $-23.89 * *$ & $-15.01 * *$ & $-17.14 * *$ & $-17.14 * *$ \\
\hline $\begin{array}{l}\text { LGG-407 } \times \text { Pusa } \\
\text { Vishal }\end{array}$ & -0.17 & $-3.47 * *$ & -2.30 & $-9.54 * *$ & $-16.04 * *$ & $11.88 * *$ & $-4.42 *$ & $-9.36 * *$ & $-18.58 * *$ & $-9.92 * *$ & $-16.53 * *$ & $-11.25 * *$ \\
\hline LGG-407 × PM-5 & $2.53 *$ & $2.96^{*}$ & $1.79 *$ & $9.78 * *$ & $8.57 * *$ & $13.95 * *$ & $14.89 * *$ & $10.46^{* *}$ & $7.52 * *$ & $9.35 * *$ & $7.73 * *$ & $14.54 * *$ \\
\hline LGG-407 x IPM-2-14 & $-14.59 * *$ & $-14.79 * *$ & $-13.35 * *$ & $-14.80 * *$ & $-18.06 * *$ & $-13.99 * *$ & -3.72 & -4.43 & $-14.16 * *$ & $-14.64 * *$ & $-15.58 * *$ & $-10.24 * *$ \\
\hline LGG-407 × PM-110 & $-4.21 * *$ & $-6.90 * *$ & $-5.78 * *$ & $-13.32 * *$ & $-17.33 * *$ & $-13.23 * *$ & $-18.41 * *$ & $-19.21 * *$ & $-27.43 * *$ & $-12.44 * *$ & $-17.11 * *$ & $-11.86^{* *}$ \\
\hline S.E.(Heterosis) & 0.28 & 0.33 & 0.33 & 0.13 & 0.15 & 0.15 & 0.01 & 0.01 & 0.01 & 0.15 & 0.18 & 0.18 \\
\hline Range Low & -14.59 & -14.79 & -14.55 & -25.50 & -27.73 & -30.91 & -22.77 & -29.20 & -30.97 & -28.00 & -30.31 & -32.43 \\
\hline High & 5.27 & 5.72 & 5.43 & 15.61 & 16.91 & 16.91 & 18.75 & 14.00 & 29.20 & 9.35 & 7.73 & 14.54 \\
\hline
\end{tabular}

RH: Relative heterosis; HB: Heterobeltiosis; SH: Standard heterosis

*Significant at 5\%level; ** Significant at $1 \%$ level 


\section{0 seed weight (g)}

Relative heterosis for this trait was found to be significant and high and ranged from 27.63 (LGG-574 $\times$ PM-110) and 34.35 (MGG-295 $\times$ PM-110). Out of 20 crosses four crosses registered significant positive relative heterosis, while nine crosses registered significant negative relative heterosis for this trait. Heterosis over better parent varied from -37.40 (LGG-574 $\times$ IPM-2-14) to 30.54 (MGG-295 $\times$ IPM-2-14). Out of 14 crosses three crosses recorded significant positive heterobeltiosis and 11 crosses showed significant negative heterobeltiosis. Estimates of heterosis over standard variety LGG-460 ranged from $-14.21(\mathrm{LGG}-460 \times \mathrm{PM}-110)$ to 42.13 (MGG-295 $\times$ PM-110) in crosses. Out of seven crosses four crosses registered significant positive standard heterosis and three crosses registered significant negative standard heterosis.

The crosses MGG-295 $\times$ PM-110 and MGG$295 \times$ IPM-2-14 displayed significant positive standard heterosis along with significant positive relative heterosis and heterobeltiosis indicating that these crosses are heterotic for this trait and could be exploited for evolving the lines with increased test weight.

\section{Seed yield per plant (g)}

The highest significant relative heterosis for seed yield per plant was registered by TM-96$2 \times$ PM-5 (57.45). The cross LGG-574 $\times$ IPM-2-14 (-22.39) recorded low significant relative heterosis. Thirteen crosses showed significant relative heterosis, out of which 12 crosses exhibited significant heterosis in positive direction, while one cross exhibited in negative direction. The range of heterobeltiosis among the crosses varied from -30.38 (LGG-574 × IPM-2-14) to 53.67 (TM96-2 $\times$ PM-5). Out of 20 crosses, 10 crosses showed significant positive heterosis over better parent and two crosses showed significant negative heterosis over better parent. Significant standard parent heterosis was registered by the cross LGG-574 $\times$ Pusa Vishal (51.04) followed by LGG-460 $\times$ Pusa Vishal (47.56), LGG-574 × PM-5 (42.76) and LGG-460 $\times$ IPM-2-14 (40.80). Out of 20 crosses, eight crosses namely TM-96-2 $\times$ PM5, TM-96-2 $\times$ IPM-2-14, LGG-574 $\times$ Pusa Vishal, LGG-574 $\times$ PM-5, LGG-460 $\times$ Pusa Vishal, LGG-460 $\times$ IPM-2-14, LGG-460 $\times$ PM-110 and LGG-407 $\times$ PM-5 registered significant positive heterosis over standard variety.

Hence, these crosses can be effectively utilized in plant breeding programmes for improving the seed yield in greengram as these crosses may yield superior segregants for seed yield in the succeeding generations.

\section{Harvest Index (\%)}

The magnitude of relative heterosis exhibited by the crosses for the harvest index varied from $-22.82($ LGG-460 $\times$ PM-5) to 22.67 (TM-96-2 $\times$ IPM-2-14). Out of 11 crosses, eight crosses displayed average heterosis in positive direction and three crosses displayed average heterosis in negative direction. Highest significant positive heterosis over better parent was recorded by the cross TM96-2 $\times$ IPM-2-14 (21.66), whereas the cross LGG-460 × PM-5 (-25.29) recorded lowest significant heterobeltiosis. Out of 20 crosses two crosses registered significant positive heterobeltiosis, while three crosses registered significant negative heterobeltiosis. The range of standard heterosis observed for the harvest index among the crosses was from -25.29 $($ LGG-460 $\times$ PM-5) to $17.30($ LGG-574 $\times$ Pusa Vishal). Four crosses exhibited significant positive heterosis over the standard variety for harvest index, whereas three crosses exhibit significant negative standard heterosis. 


\section{Total protein content (\%)}

Relative heterosis for total protein content among 20 crosses varied from -14.59 (LGG$407 \times$ IPM-2-14) to $5.27($ LGG-574 × PM-5). Out of 20 crosses, 12 crosses recorded significant negative heterosis over mid parent for total protein content, while four crosses registered significant positive heterosis. Heterobeltiosis for the total protein content varied from $-14.79(\mathrm{LGG}-407 \times \mathrm{IPM}-2-14)$ to 5.72 (LGG-574 $\times$ PM-5). Out of 20 crosses, three crosses registered significant positive heterobeltiosis and 13 crosses registered significant negative heterobeltiosis. Standard heterosis ranged from -14.55 (LGG-460 $\times$ PM-110) to 5.43 (LGG-574 $\times$ PM-5). Out of 20 crosses, only three crosses namely LGG$574 \times$ PM-5, LGG-460 $\times$ IPM-2-14 and LGG$407 \times$ PM-5 recorded significant positive standard heterosis. High magnitude of heterosis over the standard parent was exhibited by the cross LGG-574 $\times$ PM-5. The crosses, LGG-574 $\times$ PM-5, and LGG-460 $\times$ IPM-2-14 and LGG-407 $\times$ PM-5 registered significant standard heterosis along with positive relative heterosis and heterobeltosis. Hence, these crosses could be exploited as parents in hybridization programmes as they may throw desirable segregants for improvement of seed protein content.

\section{Total sugars (\%)}

Low relative heterosis was registered by the cross MGG-295 × Pusa Vishal (-25.50) and high relative heterosis was displayed by LGG-460 × IPM-2-14 (15.61). Out of 20 crosses evaluated, only four crosses TM-96-2 $\times$ PM-5, LGG-460 $\times$ Pusa Vishal, LGG-460 $\times$ IPM-2-14 and LGG-407 $\times$ PM-5 exhibited significant heterosis over mid parent. The range of heterobeltiosis observed was from 27.73 (MGG-295 $\times$ Pusa Vishal) to 16.91 (LGG-460 $\times$ IPM-2-14). Four crosses LGG$574 \times$ PM-5, LGG-460 × PM-5, LGG-460 ×
IPM-2-14 and LGG-407 × PM-5 displayed significant positive heterosis over better parent for the total sugars. The magnitude of heterosis over standard variety varied from 30.91 (MGG-295 $\times$ Pusa Vishal) to 16.91 (LGG-460 × IPM-2-14). Three crosses, LGG$460 \times$ IPM-2-14, LGG-407 $\times$ Pusa Vishal and LGG-407 $\times$ PM-5 excelled over the standard variety LGG-460 for this trait. Among the 20 crosses, LGG-460 × IPM-2-14 and LGG-407 $\times$ PM-5 registered positive significant average heterosis, heterobeltosis, and standard heterosis over the best cultivar. Hence, these crosses can be considered as heterotic cross combinations for improving the total sugar content of greengram.

\section{Reducing sugars (\%)}

High relative heterosis for reducing sugars was recorded by MGG-295 $\times$ IPM-2-14 (18.75) and the low relative heterosis was registered by MGG-295 × PM-5 (-22.77). Out of 20 crosses, nine crosses exhibited significant positive relative heterosis for this trait. The range of heterobeltiosis varied from -29.20 (LGG-460 $\times$ Pusa Vishal) to 14.00 (MGG-295 $\times$ IPM-2-14) followed by MGG$295 \times$ PM-110 (13.57). Eight crosses exhibited significant positive heterobeltiosis for reducing sugars. High standard heterosis for reducing sugars was registered by LGG$460 \times$ Pusa Vishal (29.20) and the low standard heterosis was recorded by MGG-295 $\times$ PM-5 (-30.97). The crosses viz., LGG-460 $\times$ IPM-2-14 and LGG-407 $\times$ PM-5 displayed significant standard heterosis over better variety and also showed significant positive relative heterosis and heterobeltiosis. Hence, these crosses of could be exploited for developing lines with high reducing sugars.

\section{Non- reducing sugars $(\%)$}

High relative heterosis (9.35) and heterobeltiosis (7.73) was exhibited by the 
cross LGG-407 $\times$ PM-5 and lowrelative heterosis (-28.00) and heterobeltosis (-30.31) was registered by MGG-295 × Pusa Vishal. Among the 20 crosses studied, two crosses recorded significant posistive mid parent heterosis, while only one cross registered significant positive heterosis over better parent. High standard heterosis was registered by the cross LGG-407 × PM-5 (14.54), while the low standard heterosis was recorded by MGG-295 × Pusa Vishal (-32.43). Four crosses displayed significant positive heterosis over the standard variety for this trait. In this study, the cross LGG-407 $\times$ PM-5 registered high relative heterosis and heterobeltiosis for non-reducing sugars also showed significant positive heterosis over standard variety. Hence, this cross can be exploited in breeding programmes to obtain desirable segregants for non-reducing sugars.

The above results revealed that the cross LGG-574 $\times$ PM-5 showed significant standard heterosis for branches per plant, clusters per plant, seed yield per plant, 100 seed weight, harvest index, total protein content and non-reducing sugar while the cross LGG-460 $\times$ IPM-2-14 recorded significant heterosis for plant height, branches per plant, clusters per plant, pods per cluster, seeds per pod, seed yield per plant, total protein content, total sugars, reducing sugar and non-reducing sugars. Further, the crosses TM-96-2 × IPM-2-14 exhibited significant standard heterosis for clusters per plant, pods per cluster and harvest index; LGG-460 $\times$ Pusa Vishal recorded desirable standard heterosis for branches per plant, clusters per plant, seeds per pod, harvest index and reducing sugar, LGG-460 × PM-110 showed significant standard heterosis for clusters per plant and seed yield per plant.

Similarly, cross LGG-574 $\times$ Pusa Vishal showed significant standard heterosis for branches per plant, clusters per plant, pods per cluster, seeds per pod, 100 seed weight and harvest index; LGG-407 $\times$ Pusa Vishal for total sugars and pods per cluster; LGG-407 $\times$ PM-5 for days to maturity, plant height, clusters per plant, seed yield per plant, total protein content,, total sugars, reducing sugar and non-reducing sugar; LGG-407 × IPM2-14 for clusters per plant; TM-96-2× PM-110 for branches per plant and clusters per plant; MGG-295 × Pusa Vishal for clusters per plant and plant height; LGG-460 × PM-5 for clusters per plant; LGG-407×Pusa Vishal for pods per cluster, MGG-295 × IPM-2-14 andMGG-295 × PM-110for seeds per pod and100 seed weight recorded significant positive standard heterosis. These results are in conformity with those of Aher et al., (2000b) for seed yield, Joseph and Santhoshkumar (2000) for plant height, number of branches per plant, pods per plant, test weight and seed yield; Loganathan et al., (2001b) for number of seeds per pod; Sunil and Prakash (2011) for number of branches per plant, pods per plant, test weight and seed yield per plant and srivastava and Singh (2013) for seed yield per plant.

It was evident from the present study that significant and positive heterosis for seed yield could be due to the heterosis of number of branches per plant, number for clusters per plant, number pods per cluster and seeds per pod and harvest index. This denotes that the heterosis for seed yield was through heterosis for its components traits as suggested by Grafius (1959). The crosses LGG-574 × Pusa Vishal, LGG-574 × PM-5, LGG-460 × Pusa Vishal, LGG-460 $\times$ IPM-2-14 and LGG-407 $\times$ PM-5 were identified as promising crosses for seed yield, its components and nutritional traits. These crosses could exploited for deriving high yielding lines in greengram. Though exploitation of heterosis is limited in greengram due its autogamous nature and lack of feasibility of large scale production of hybrids, the identified superior crosses could 
be exploited for obtaining superior desirable segregants in subsequent generations through biparental mating followed by selection.

\section{References}

Aher, R. P., Surve, P. P. and Dahat, D. V. (2000). Agric. Sci. Dig. 20(1): 5.

Barad, H. R., Pithia, M. S. and Vachhani, J. H. (2008). Legume Res. 31(1): 68.

Datta, S., Gangwar, S., Shiv Kumar, Gupta, S., Rai, R., Kaashyap, M., Singh, P., Chaturvedi, S.K., Singh, B.B. and Nadarajan, N. 2012. Genetic diversity in selected Indian greengram [Vigna radiata (L.) Wilczek] cultivars using RAPD markers. American Journal of Plant Sciences. 3: 1085-1091.

Grafius, J. E. (1959). Agron. J., 5: 551.
Joseph, J and Santhosh kumar, A.V. (2000). Res. Crops. 2: 221.

Loganathan, P., Saravanan, K., Thangavel, P and Ganesan, J. (2001). Legume Res. 24(2): 77.

Marappa, N. (2008). Asian J. Bio Sci. 3(2): 289.

Panse, V.G. and Sukhatme, P.V. 1985. Statistical methods for Agricultural workers, Indian Council of Agricultural Research, New Delhi.

Rai, B. (1979). Heterosis breeding. Agro Biological Publications, New Delhi. pp: 155.

Srivastava, R.L and Singh, G. (2013). Indian J. Res., 4(1): 131. Sunilkumar, B and Prakash, M. (2011). Karnataka J. Agri. Sci., 24(4): 523.

\section{How to cite this article:}

Kalpana, S., N.V. Naidu and Reddy, D.M. 2018. Heterosis for Yield, Yield Components and Nutritional Traits in Mung Bean (Vigna radiata (L.) Wilczek). Int.J.Curr.Microbiol.App.Sci. 7(11): 1780-1791. doi: https://doi.org/10.20546/ijcmas.2018.711.203 